\section{High-Resolution Semi- Quantitative Real-Time PCR without the Use of a Standard Curve}

BioTechniques 31:502-508 (September 2001)

\begin{abstract}
The repeatability and sensitivity of a simple, adaptable, semi-quantitative, realtime RT-PCR assay was investigated. The assay can be easily and rapidly applied to quantitate relative levels of any gene product without using standards, provided that amplification conditions are specific for the $P C R$ product of interest. Using the LightCycler ${ }^{\mathrm{TM}}$ real-time PCR machine, a serial 10-fold dilution series (spanning four orders of magnitude) of a 379-bp cDNA tem plate was amplified, and the PCR product was detected using $S Y B R^{\circledR}$ Green I chem istry. The experiment was repeated on a subsequent day. The experimental design was such that the data lent itself to analysis using an appropriate method for testing repeatability. It was found that, within a single assay, for samples assayed in triplicate, a difference of $23 \%$ may be reliably detected. Furthermore, when all of the factors that contribute to variability in the assay are taken into account, such as day-to-day variation in pipetting and amplification efficiency, a 52\% difference in target template can be detected using a sample size of 4 . The assay was found to be linear over at least four orders of magnitude.
\end{abstract}

\section{INTRODUCTION}

With the advent of sophisticated PCR machines that incorporate software-driven fluorescence analysis systems, real-time PCR (5) is rapidly becoming a more common method for the analysis and quantification of gene expression. Indeed, the data output from certain real-time PCR machines gives an immediate appreciation of the kinetics of the PCR occurring within the tube and, in addition, gives an instantaneous visual representation of the amount of PCR product present follow ing each cycle. Following a single real- time PCR, the data extracted give the type of information that was only previously inferable from multiple conventional PCRs.

Real-time PCR data collection may be facilitated through the use of either the fluorescent dsDNA binding dye, SYBR ${ }^{\circledR}$ Green I (Roche Molecular Biochemicals, Mannheim, Germany) (7), or a number of complex chemistries that use short, fluorescent-tagged oligonucleotide probes that bind within the PCR product. The advantages and disadvantages of each of these detection chemistries have been described elsewhere (9); however, the use of SYBR Green I in a real-time PCR assay permits it to be easily applied to the detection of any PCR product for which reaction conditions can be optimized.

Despite the obvious importance of obtaining quantitative information regarding input template to a PCR, in the vast majority of cases it is simply desirable to determine whether a treatment or condition results in an increase or decrease in template relative to a control sample (3). In such cases, it is sufficient to determine the fold difference or percentage change in one sample relative to another. Here, we report on a rapid, adaptable, and high-resolution PCR assay for the semi-quantitative analysis of PCR product using the LightCycler ${ }^{\mathrm{TM}}$ real-time PCR instrument (Roche Molecular Biochemicals) and SYBR Green I chemistry. The data analysis is performed in such a fashion that it circum vents the need for a standard curve, thus saving on reagents and allowing more samples to be assayed in each run.

\section{MATERIALS AND METHODS}

\section{Experimental Aims}

This experiment was designed to answer three questions about a semiquantitative real-time PCR method. First, we wished to determine the intraassay variability and, hence, the minimum difference that could be differentiated between sets of replicate samples. Second, we wished to determine the minimum difference between groups of paired treated and control samples that could be detected by this technique, taking into account factors such as pipetting and amplification efficiency that influence both inter- and intra-assay variability. Third, we wished to determine the dynamic range over which the machine could provide this sensitivity and to confirm that the machine provided an accurate representation of the amount of input template over this range.

\section{Experimental Procedure}

The PCR template used was TIMP1 cDNA, generated from mRNA that had been extracted from the sclera of the tree shrew, a South East Asian arboreal mammal. Primers directed against the human sequence of TIMP-1 were first used to generate a product. This product was purified and sequenced; its identity was confirmed against the known human sequence and "nested" species-specific primers designed (product size $379 \mathrm{bp}$ ). These specific primers were used to generate template cDNA, which was then purified from a single band on a gel. The cDNA was quantified using spectrophotometry, and a 10-fold dilution series was set up.

The amplification conditions of the TIMP-1 template were optimized for the LightCycler instrument and the subsequent PCR runs showed a single PCR product during melting curve and electrophoretic analysis. PCR amplifications were performed using the FastStart ${ }^{\mathrm{TM}}$ DNA Master SYBR Green I (Roche Molecular Biochemicals) realtime PCR kit. Primers ( $1 \mu \mathrm{M}$ each), additional magnesium chloride (final concentration $3 \mathrm{mM})$, and template $(2 \mu \mathrm{L}$ of the appropriate dilution) were combined with $2 \mu \mathrm{L}$ FastStart reaction mixture (containing FastStart Taq DNA polymerase, SYBR Green I dye, dNTPs, magnesium chloride, and reaction buffer) and PCR-grade water to a volume of $20 \mu \mathrm{L}$.

The reaction series consisted of six samples from each of the neat $(2.5$ $\mathrm{pg} / \mu \mathrm{L}$ ) and four serial 10 -fold dilutions (30 samples in total). For the purposes of our analysis and to ensure randomization of the data, it was predetermined that, within each of the dilutions, sample 1 would be compared with sam ple 2 , sample 3 with sample 4 , and sample 5 with sample 6 . Furthermore, it was predetermined that samples 1,2 , 


\section{Short Technical Reports}

and 3 would constitute a triplicate, as would samples 4, 5, and 6. Finally, each of the triplicates at each dilution series was paired with one of the neat sample triplicates, and these combinations were allied to similar combinations in an experiment carried out on a subsequent day.

A main master mixture was produced containing primers, magnesium chloride, FastStart mixture, and water. This main mixture was subdivided into five separate aliquots, to which one of the neat or 10 -fold dilution series template was added. The glass capillary reaction tubes of the LightCycler were loaded with $20 \mu \mathrm{L}$ each from these aliquots, as described above, and the run was initiated. The cycling conditions were as follows: denaturation for $10 \mathrm{~min}$ at $95^{\circ} \mathrm{C}$; amplification for 40 cycles, with denaturation for $0 \mathrm{~s}$ at $95^{\circ} \mathrm{C}$, annealing for $10 \mathrm{~s}$ at $60^{\circ} \mathrm{C}$, extension for $16 \mathrm{~s}$ at $72^{\circ} \mathrm{C}$, and detection after $2 \mathrm{~s}$ at $87^{\circ} \mathrm{C}$. A melting curve analysis was performed from $65^{\circ} \mathrm{C}$ to $95^{\circ} \mathrm{C}$. This exact experimental protocol was repeated on a subsequent day from newly produced master mixtures.

\section{Data Analysis}

The experiment we report here was designed to allow data to be analyzed using the method described by Altman and Bland (1). This type of analysis is one of the more stringent methods for assessing the agreement between two experimental methods and can also be adapted to investigate the repeatability of a technique.

Raw data (fluorescence vs. cycle number) from the LightCycler was used to enable the calculation of amplification efficiency, using GraphPad Prism $^{\mathrm{TM}}$ software (GraphPad Software, San Diego, CA, USA). Data were exported as a tab-delineated text file using the Export function in the LightCycler software. The text file was opened as a worksheet in Microsoft ${ }^{\circledR}$ Excel $^{\circledR}$, and the appropriate data were copied and pasted into a GraphPad Prism spreadsheet. Linear regression analysis was performed on log fluorescence data to indicate the log linear phase of the reaction and to determine the amplification efficiency (the inverse log of the slope in the log linear phase). To determine

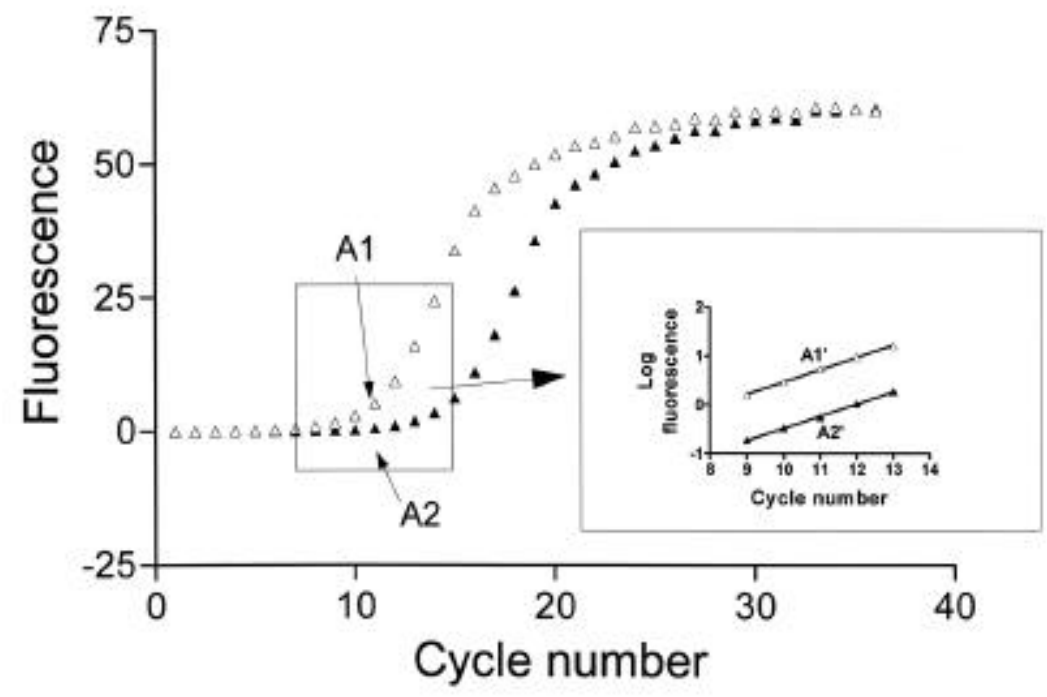

Figure 1. Representative real-time amplification profile of two samples illustrating a 10-fold difference in starting template. The main figure shows fluorescence against cycle number (raw data). The box indicates the log linear phase of the reaction, and the inset graph shows the log linear phase of the reaction plotted as log fluorescence against cycle number. The two points indicated on the main figure (A1 and A2) are within the log linear phase of the reaction and separated by a fold difference of A1/A2 (10fold). The log of the fold difference is represented by the difference between the corresponding points $\left(\mathrm{A} 1^{\prime}-\mathrm{A} 2^{\prime}\right)$ on the inset figure (as shown by $\left.\log (\mathrm{A} 1 / \mathrm{A} 2)=\log \mathrm{A} 1-\log \mathrm{A} 2=\mathrm{A} 1^{\prime}-\mathrm{A} 2^{\prime}\right)$. As a result, the fold difference can be calculated from the expression $10\left(\mathrm{Al}^{\prime}-\mathrm{A} 2^{\prime}\right)(10$-fold $)$.

intra-assay variability, crossing point data (cycle number) from the LightCycler Summary Report was entered into an Excel spreadsheet. The crossing point is the calculated cycle number at which fluorescence in the reaction cuts an arbitrary baseline (defined by fluorescence noise) while the reaction is in the log-linear phase. Predetermined paired sample data within each experiment were subtracted from each other (sample 1 - sample 2, sample 3 - sample 4 , etc.), and the differences were plotted against the log of the sample dilution. Sample statistics (mean and standard error), power, and sample size calculations ( 1 - sample $t$-test power calculation) were performed using Minitab $^{\mathrm{TM}}$ software (Minitab, State College, PA, USA); the calculated efficiency of reactions was then used to convert the appropriate values to fold or percentage differences using the equation $\mathrm{F}=\mathrm{E}^{n}(\mathrm{~F}=$ fold difference, $\mathrm{E}=$ amplification efficiency, and $n=$ number of cycles between crossing points).

For the purposes of testing the inter(or total) assay variability, we considered the predetermined triplicate data from the dilution series as "treated" samples and the neat data as "control" samples. Raw data were extracted from the LightCycler and pasted into a GraphPad Prism spreadsheet as described above. GraphPad Prism software was used to perform linear regression analysis on the mean of the raw $\log$ fluorescence data from paired treated and control triplicates. It can be shown that the vertical distance between the treated and control lines, in the log linear phase of the reaction, is the log of the fold difference between the samples and that the gradient of the lines represents the log of the efficiency of the PCR (Figure 1). This method not only allowed us to quickly determine the $\log$ of the fold difference between samples but also to statistically test that the mean amplification efficiency of the two sets of samples was identical (i.e., the lines were parallel), a condition that must be met for any comparison to be valid. GraphPad Prism performs this analysis using the method described by Zar (10). To determine the total assay variability, the fold differences between treated and control samples for predetermined paired triplicates were sub- 
tracted from each other and plotted against the $\log$ dilution factor. As above, sample statistics, power, and sample size calculations were performed using Minitab software. A number of graphing/statistical packages will be capable of the manipulations and statistical analyses described above; however, the authors would be happy to provide a template of the GraphPad Prism spreadsheet used in the current study.

\section{RESULTS}

\section{Amplification Efficiency}

Data analysis showed that in all experiments the mean amplification efficiency was 1.81 , and there was no significant difference in amplification efficiency between any of the comparisons performed between treated and control samples $(P>0.05$ in all cases $)$.

\section{Intra-Assay Variability}

Intra-assay analysis of crossing point data using the method of Altman and Bland (1) is shown in Figure 2A. The mean difference between paired observations was not significantly different from zero $(P=0.43)$, indicating that the intra-assay variability was random, as expected, and not systematic. As there was no apparent difference in variability at any dilution, these values were pooled, and the sample statistics were calculated (mean $=0.016, \mathrm{SD}=$ 0.109 , and $n=29$ ). Application of this data to a power statistic indicated that, for a power of 0.8 , triplicate measurements would allow a 0.36 cycle difference in crossing point (corresponding to a $23 \%$ difference when corrected for the amplification efficiency) between samples to be detected at a significance level of 0.05 .

\section{Inter- (or Total) Assay Variability}

Semi-quantitative analysis is based on the precept that a treated sample is expressed as a value relative to its control sample (3). This value may be either a fold or percentage difference. For the purposes of testing the inter- (or total) assay variability, we considered the predetermined triplicate data from the dilution series as treated samples and the neat data as control samples. Consequently, the mean difference between predetermined treated and control triplicates was expressed as a fold difference in each of the two experiments. The corresponding fold differences in experiment 2 were then taken from those in experiment 1 and plotted against the log of the dilution factor, in accordance with the analysis of Altman and Bland (1), to demonstrate inter- (or total) assay variability (Figure 2B). Once again, the mean of these values was not significantly different from zero $(P=0.47$, mean $=0.023, \mathrm{SD}=$ 0.086 , and $n=8)$. Again, there was no apparent difference in variability at any dilution and data was pooled and applied to a power calculation. It was found that a mean difference of $52 \%$ between treated and control conditions can be determined with 0.05 confidence using triplicates of treated and control samples and an overall sample size of 4 . Furthermore, a sample size of 5 will allow discrimination of a $39 \%$ difference at a significance level of 0.05 .

\section{Dynamic Range}

The mean fold difference between the predetermined treated and control triplicates at each dilution series was used to calculate the dilution factor. The $\log$ of this calculated value was plotted against the $\log$ of the actual

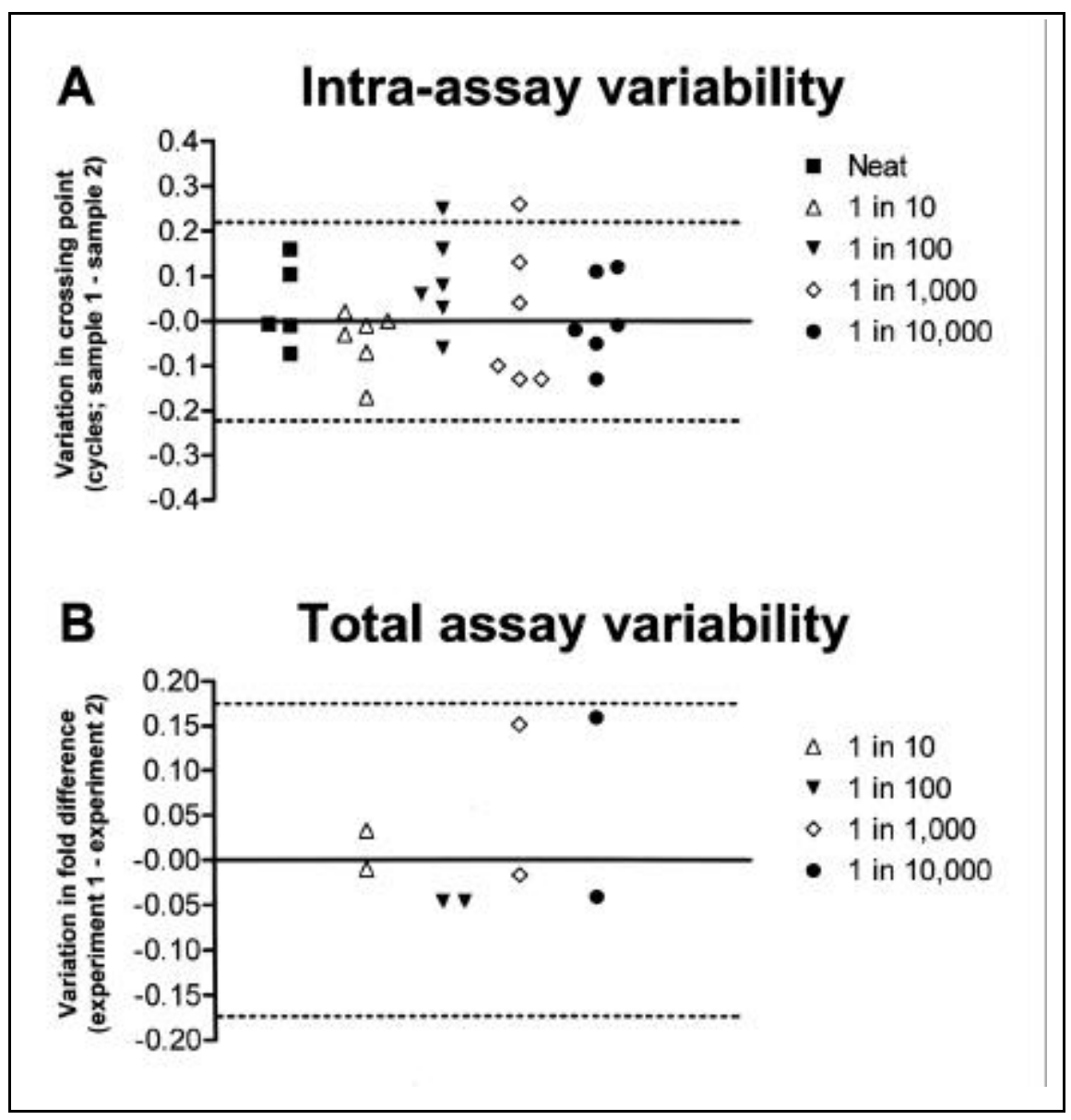

Figure 2. Variability of semi-quantitative real-time PCR assay. (A) Graphical representation of intraassay variability as calculated using the method of Altman and Bland (1). Difference in crossing point between sample 1 and sample 2 of a predetermined duplicate are plotted against the dilution factor of the samples. (B) Graphical representation of total assay variability when the mean difference between treated and control triplicates from separate assays were compared using the method of Altman and Bland (1). Fold differences between predetermined treated and control triplicates in two separate experiments were subtracted from each other (experiment 1 - experiment 2 ) and plotted against the dilution factor. The dotted lines indicate \pm 2 SD from the mean. 
dilution to indicate the reliability of the assay in estimating the input amount of template (Figure 3). That the linear regression through these points has a gradient of $0.99 \pm 0.01$ (SEM) reinforces the obvious precision of this assay at up to a $4 \log$ unit (10000-fold) difference between treated and control conditions.

\section{DISCUSSION}

This study has demonstrated the high-resolution capabilities of a realtime PCR machine in performing a simple, adaptable, semi-quantitative assay. The data clearly shows that a $23 \%$ difference between samples may be reliably detected, without the use of a standard curve, if triplicates are employed. Furthermore, despite the small number of observations, a sample size of 4 is sufficient to allow detection of at least a $50 \%$ (1.5-fold) difference in PCR template quantity in an experimental system. Semi-quantitative data obtained from this assay is reliable over at least four orders of magnitude. In an experimental paradigm, this methodology can easily be applied to the measurement of a housekeeping gene, thus allowing adjustment to be made for any variation in the efficiency of mRNA extraction and reverse transcription.
The presence of a PCR inhibitor or differences in template quality between samples may affect amplification efficiency, which may produce different efficiencies between treated and control samples. In such an instance, any quantitative or semi-quantitative comparison between samples is invalidated. One of the strengths of the method of data analysis reported here is that it immediately alerts the investigator to a problem of this nature. In such an instance, it is essential that an internal standard and/or a validated housekeeping gene be also assessed.

The levels of sensitivity reported here compare favorably with the current benchmark in gene expression studies, competitive RT-PCR. In the literature, studies employing competitive RT-PCR generally report assay variability by quoting the coefficient of variation $(\mathrm{CV} \%=(\mathrm{SD} / \mathrm{mean}) \times 100)$. The CV of the assay reported here was $2.5 \%$ (total assay variability), which compares favorably with previously reported values for competitive RT-PCR ranging from $6.6 \%$ to $38.2 \%(4,8)$. $\mathrm{Re}$ cently, investigators developing a semiquantitative real-time PCR assay using SYBR Green I and an external standard curve reported a standard deviation (referred to as the "inter-assay variability") for their measured values of less

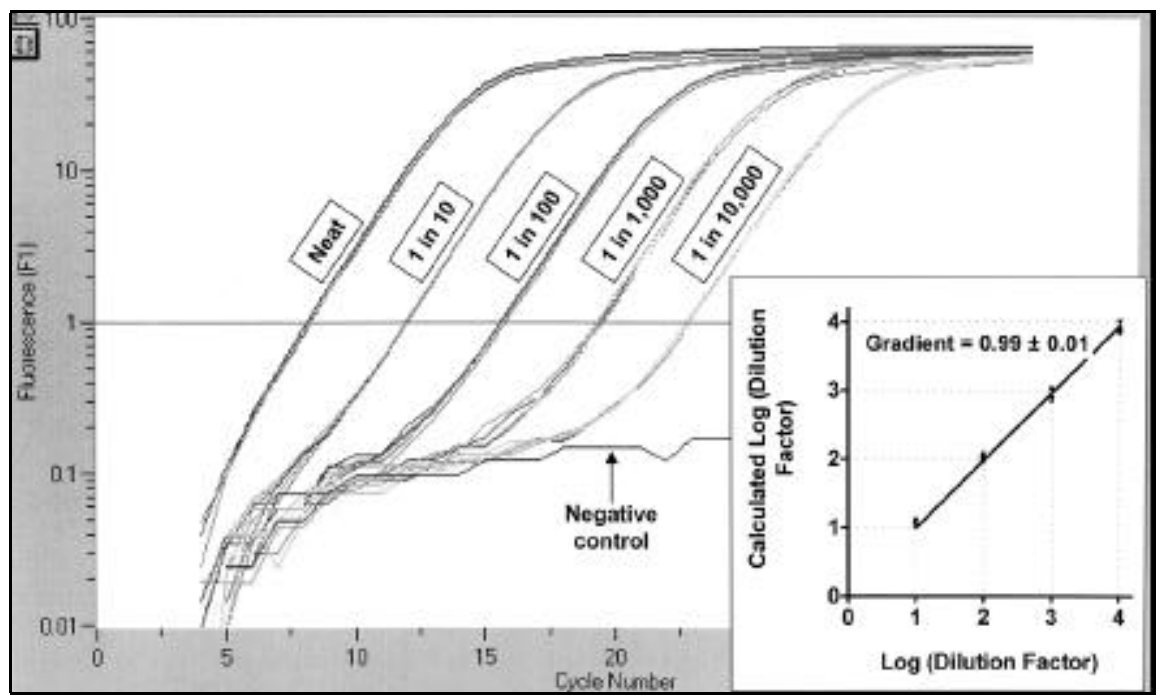

Figure 3. LightCycler output and inset graphical representation to show the linearity of the assay over four orders of magnitude. The main figure shows the output from the LightCycler (log fluorescence against cycle number) indicating the crossing point of triplicates from a 10 -fold dilution series. The inset figure represents the calculated log dilution factor (obtained from comparing each dilution triplicate to the neat sample and calculating the dilution factor) against the actual dilution factor. The line represents a linear regression through the mean of the four points at each dilution. 
than $0.06 \log$ units (2). The equivalent value for the assay reported here was found to be $0.058 \log$ units, which is unsurprising given that an identical machine and detection method were employed in both assays.

In the present study, the dynamic range of the assay was tested over four orders of magnitude; however, the LightCycler instrument has previously been shown to linearly amplify target template over 7-8 orders of magnitude $(2,6,9)$. Investigators reported that linear amplification was obtainable from a maximum of 10-20 ng target tem plate $/ 5 \mu \mathrm{L}$ reaction volume down to $10^{2}$ copies of target template (9). Furthermore, it has been reported that there is no difference in the variability of the calculated target template concentration from $10^{7}$ to $10^{3}$ copies (2), as was found in the present study. The maximum template amount used in the present study ( $5 \mathrm{pg} / 20 \mu \mathrm{L}$ reaction) was the equivalent of $1.25 \mathrm{pg} / 5 \mu \mathrm{L}$ reaction volume, suggesting that the present assay should remain linear for another three to four orders of magnitude, were target template concentration to be increased. In addition, the minimum amount of target template used $(0.5 \mathrm{fg})$ was calculated to represent $2.67 \times 10^{3}$ copies of template, also suggesting that the assay should remain linear for another order of magnitude, were target template concentration to be decreased. Amounts of target template less than $10^{2}$ copies would be expected to produce increased variability. As a result, we conclude that the resolution and linearity of this assay should hold over eight orders of magnitude.

The findings of the current study suggest that the semi-quantitative real-time PCR assay described in this report is at least as sensitive as any other previously reported RT-PCR-based assay. Furthermore, this assay can be conducted relatively cheaply and without the use of any external or internal standards.

\section{REFERENCES}

1.Altman, D.G. and J.M. Bland. 1983. Measurement in medicine: the analysis of method comparison studies. Statistician 32:307-317.

2.Dumoulin, F.L., H.D. Nischalke, L. Leifeld, A. von dem Bussche, J.K. Rockstroh, T. Sauerbruch, and U. Spengler. 2000. Semi- quantification of human C-C chemokine $\mathrm{mR}$ NAs with reverse transcription/real-time PCR using multi-specific standards. J. Immunol. Methods 241:109-119.

3.Ferre, F. 1992. Quantitative or semi-quantitative PCR: reality versus myth. PCR Methods Appl. 2:1-9.

4.Giguere, S. and J.F. Prescott. 1999. Quantitation of equine cytokine mRNA expression by reverse transcription-competitive polymerase chain reaction. Vet. Immunol. Im munopathol. 67:1-15.

5.Higuchi, R., C. Fockler, G. Dollinger, and R. Watson. 1993. Kinetic PCR analysis: realtime monitoring of DNA amplification reactions. Biotechnology 11:1026-1030.

6.Morrison, T.B., J.J. Weis, and C.T. Wittwer. 1998. Quantification of low-copy transcripts by continuous SYBR Green I monitoring during amplification. BioTechniques 24:954-962.

7.Skeidsvoll, J. and P.M. Ueland. 1995. Analysis of double-stranded DNA by capillary electrophoresis with laser-induced fluorescence detection using the monomeric dye SYBR Green I. Anal. Biochem. 231:359-365.

8.Venturi, G., R. Ferruzzi, L. Romano, M. Catucci, P.E. Valensin, and M. Zazzi. 2000 Ultrasensitive in-house reverse transcriptioncompetitive PCR for quantitation of HIV-1 RNA in plasma. J. Virol. Methods 87:91-97.

9.Wittwer, C.T., M.G. Herrmann, A.A. Moss, and R.P. Rasmussen. 1997. Continuous fluorescence monitoring of rapid cycle DNA am plification. BioTechniques 22:130-138.

10.Zar, J.H. 1984. Biostatistical Analysis, 2nd ed. Prentice-Hall, Upper Saddle River, NJ.

This work was funded by a National Health and Medical Research Council (NH\&MRC) of Australia equipment grant (no. 001609) and NH\&MRC project grant no. 980677 to Neville A. McBrien. Address correspondence to Dr. Neville A. McBrien, Department of Optometry and Vision Sciences, The University of Melbourne, Victoria 3010, Australia.e-mail: n.mcbrien@optom etry.unimelb.edu.au

Received 7 November 2000; accepted 28 February 2001.

\section{Alex Gentle, Frank Anastaso- poulos, and Neville A. McBrien The University of Melbourne Victoria, Australia}

